\title{
Die Ablation des Trabekelwerks
}

\author{
M. Vogel ${ }^{\prime}, G$. Scheurer $^{\prime}, W$. Neu $^{2}, M$. Dressel $^{2}, H$. Gerhardt $^{2}$ \\ 'Abteilung Augenheilkunde, Univ.-Augenklinik Göttingen (Leiter: Prof. Dr. M. Vogel) \\ ${ }^{2}$ Laser Laboratorium, Gottingen e. V. (Leiter: Dr. H. Gerhardt)
}

Herrn Professor Dr. Rolf Marquardt zum 65. Geburtsiag gewidmet

\section{Zusammenfassung}

An enukleierten Augen führten wir experimentelle Untersuchungen zur $A$ blation des Trabekelwerks (AT) durch. Eine Quarzfaser wurde durch die Vorderkammer bis zum gegenüberliegenden Trabekelwerk geführt und dieses mit einem Excimer Laser mit 248 und $308 \mathrm{~nm}$ bestrahlt. Es reichte eine Energie von 0,3-1,5 mJ, um eine offene Verbindung zwischen Vorderkammer und Schlemmschem Kanal herzustellen. Die histologische Untersuchung der Bulbi bestätigte die klinische Beobachtung einer offenen Verbindung zwischen Vorderkammer und Schlemmschem Kanal. Dabei wurde keine nennenswerte Hitzewirkung an den abladierten Geweben festgestellt. Auf die genannte Weise konnten wir auch Cyclodialysen und basale Iridektomien durchführen. Die Durchführung der Operation ist einfach und sehr treffsicher.

\section{Ablation of Trabecular Meshwork}

In an experimental investigation we examined the possibility to create an open pathway between the anterior chamber and Schlemm's canal by excimer laser ablation of the trabecular meshwork (AT) in enucleated eyes. A quartzfiber was directed through the anterior chamber to the opposite chamber angle. With an energy of $0.3-1.5 \mathrm{~mJ}$ and wavelenghts of 248 and $308 \mathrm{~nm}$ pores were easily made into the trabecular meshwork leading to a direct connection between the anterior chamber and Schlemm's canal. This result was confirmed by histologic examination of the globes. The trabecular meshwork has disappeared completely. The surrounding tissue reveals only minimal thermal effects due to the laser burns. With the same method cyclodialysis and basal iridectomy may be performed. The operation is simple and effects can be placed with great accuracy.

\section{Einfiihrung in die Methodik}

Die von Krasnov (1973) und Hager (1973) in die Augenheilkunde eingeführte Lasertrabekulopunktur bestach durch die Einfachheit ihres Konzepts der Glaukomtherapie. Mit einem Laser - im Falle von Krasnov ei-

Klin. Mbl. Augenheilk, 197 (1990) 250-253

(C) 1990 F. Enke Verlag Stuttgart nem Q-switched-Kurzzeitlaser und im Falle von Hager mit einem kontinuierlich arbeitenden Argon-Laser - sollte das Trabekelwerk punktiert werden. Die Ergebnisse dieser Therapie erwiesen sich auf die Dauer jedoch als unbefriedigend. Krasnov berichtete über 10 ausgewählte Patienten, die er 1970 mit dem Laser behandelt und über 2 Jahre kontrolliert hatte. Bei 9 Patienten erreichte er eine vorübergehende Drucksenkung, die aber nur 2 bis 8 Wochen anhielt.

Bei Augen mit malignem Melanom führten wir 24 und 48 Stunden vor der Enukleation eine Lasertrabekulopunktur nach Hager mit einem Argon-Laser durch (Vogel und Schildberg 1973). Die Augen wurden nach der Enukleation histologisch untersucht. Dabei zeigte sich, daß bei Laserbehandlungen unterschiedlicher Dauer und unterschiedlicher Energie eine Koagulation des Trabekelwerks eintrat, die zu einem Verbacken der Trabekelbälkchen führte. In keinem Fall konnte der Schlemmsche Kanal eröffnet werden. Mit dieser Methode konnte offensichtlich nicht der gewünschte Erfolg erzielt werden.

Wise und Witter (1979) entwickelten dann die Methode der Lasertrabekuloplastik, indem sie bei minimaler Blende und sehr kurzer Belichtung nur zarte oberflächliche Koagulationseffekte auf das Trabekelwerk setzten und damit eine Schrumpfung der obersten Trabekellagen erreichten. Diese Methode ist heute als eine adjuvante Therapie beim Glaucoma chronium simplex anerkannt, obwohl ein dauerhafter drucksenkender Effekt, wie wir in einer prospektiven klinischen Studie (Vogel et al. 1988) nachgewiesen haben, nicht verläßlich zu erreichen ist.

In unserer Studie (Vogel und Schildberg 1973) hatten wir festgehalten, daß es wünschenswert wäre, ein Gerät mit hohen Energien im Nanosekundenbereich zur Verfügung zu haben, das in der Lage ist, das Trabekelwerk bis zum Schlemmschen Kanal zu eröffnen, ohne daß es dabei zu einer Hitzekoagulation des Trabekelwerks, des Schlemmschen Kanals oder des umgebenden Gewebes kommt. Auf diese Weise hofften wir, eine offene Verbindung zwischen Vorderkammer und Schlemmschem Kanal zu erreichen. Nach Bill et al. (1989) liegen über $90 \%$ des Abflußwiderstandes zwischen Vorderkammer und Schlemmschem Kanal. Es erscheint uns deshalb sinnvoller, hier einen Durchfluß zu schaffen, als eine fistulierende Operation vorzunehmen.

Unseres Wissens ist bisher noch nicht der Versuch unternommen worden, mit einem Excimer-Laser und einer Quarzfaser das Trabekelwerk selektiv zu entfernen und eine Verbindung zum Schlemmschen Kanal herzustellen. 


\section{Material und Methode}

Zunächst haben wir 3 Schweineaugen mit einer Saugkalotte fixiert und dann unter dem Operationsmikroskop eine Infusion in die Vorderkammer gelegt. Bei eingeschaltetem Excimer-Laser (Lambda Physik, Göttingen) mit einer Wellenlänge von 248 Nanometer wurde eine Quarzfaser von $240 \mu \mathrm{m}$ (Ensign-Bickford) langsam durch die Hornhaut geschoben.

Die Quarzfaser wurde dann quer durch die Vorderkammer zur gegenüberliegenden Kammerwinkelregion geführt und hier mit $0,35-0,8 \mathrm{~mJ}$, unter Benutzung eines HeliumNeon-Lasers als Zielstrahl, der Kammerwinkel abladiert. Dabei traten einzelne, winzige Bläschen im Kammerwasser auf. Die Augen wurden sodann in Formalin fixiert, in Paraffin eingebettet und in 400 Serienschnitte geschnitten und mit Hämatoxylin-Eosin gefärbt. Dabei zeigte sich, daß infolge der vom menschlichen Auge doch recht unterschiedlichen Kammerwinkelstruktur des Schweineauges eine gezielte Koagulation des Trabekelwerks nicht möglich war. Man konnte aber deutlich die Ablation des Gewebes im Bereich des Kammerwinkels erkennen.

Wir griffen dann auf 7 Leichenaugen zurück, die wir in ähnlicher Weise behandelten. Wir setzten eine Wellenlänge von $308 \mathrm{~nm}$ ein, die uns erlaubte, lange Fasern der Firma Ceram Optec von $200 \mu \mathrm{m}$ Durchmesser zu benutzen. Die Fasern wurden in ein von uns hergestelltes Handstück integriert, womit eine sichere Manipulation im Auge gewährleistet war. Beim Skleradurchtritt verwendeten wir Energien zwischen 1 und $5 \mathrm{~mJ}$, der Kammerwinkel wurde mit $0,7-1,5 \mathrm{~mJ}$ behandelt. Wegen der ge-

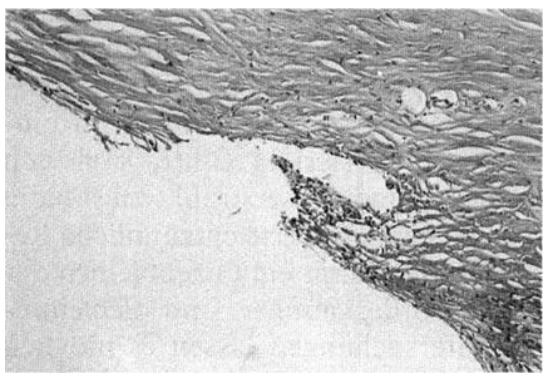

Abb. 1 Die vordere Hälfte des Trabekelwerks ist verschwunden. Es besteht eine offene Verbindung zwischen Vorderkammer und Schlemmschem Kanal. Ein thermischer Effekt ist nicht eingetreten. Es wurde mit einer Wellenlänge von $248 \mathrm{~nm}$, einer Pulslänge von 20 ns und einer Energie von $0,5 \mathrm{~mJ}$ gearbeitet. (Hämatoxylin-Eosin, $40 \times$ )

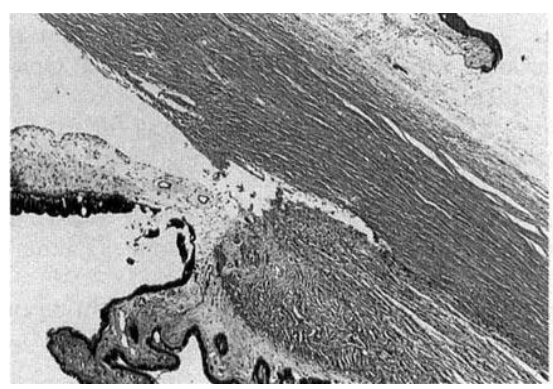

Abb. 2 Die Quarzfaser wurde hier zu welt in den Kammer winkel vorgeschoben und fuhrte zu einer tunnelartigen Tren nung des Ciliarkörpers von der Sklera im Sinne einer Cyclodialyse. In dem Tunnel befindet sich etwas Zelldetritus. Es wurde mit einer Wellenlänge von $308 \mathrm{~nm}$, Pulslänge $20 \mathrm{~ns}$ und $0,75-1,0 \mathrm{~mJ}$ gearbeitet. (Hämatoxylin-Eosin, 16x) trübten Hornhaut konnten wir auch nach Abrasion des Hornhautepithels den Kammerwinkel nicht klar einsehen und hatten Schwierigkeiten, das Trabekelwerk in der von uns gewünschten Weise zll abladieren. Entsprechend dem Lichtpunkt des HeliumNeon-Lasers, der sich außen auf der Sklera im Bereich des Kammerwinkels abbildete, wurden dann Ablationen unterschiedlicher Dauer und mit unterschiedlicher Energie durchgeführt.

Die histologischen Untersuchungen zeigen in einem Fall eine eindeutige Eröffnung alleine des Trabekelwerks mit Eröffnung des Schlemmschen Kanals zur Vorderkammer (Abb. 1). Es ist keine Koagulation des Trabekelwerks eingetreten. Das Trabekelwerk ist an dieser Stelle verschwunden.

In anderen Augen war die Quaszfaser nicht genau auf das Trabekelwerk gerichtet, weil der Einblick in den Kammerwinkel nicht möglich war. Man erkennt hier Ablationen im Sinne einer Cyclodialyse (Abb. 2 und 2a). In einem anderen Fall ist es zu einer Ablation der Iriswurzel nach Art einer basalen Iridektomie gekommen (Abb. 3). Nur in einem sehr schmalen Berejch in unmittelbarer Nachbarschaft der Quarzfaser ist eine umschriebene Basophilie des Gewebes als Ausdruck eines minimalen umschriebenen Wärmeeffektes entstanden. In zwei Fällen konnten wir ein frisch enukleiertes Auge verwenden. Die Hornhaut war ausreichend klar, so daß die Quarzfaser genau in den Bereich des Trabekelwerks vorgeschoben werden konnte. Die Excimer-Laserkoagulation erfolgte bei $0,5-1,5 \mathrm{~mJ}$ und einer Pulslänge von 20 Nanosekunden und führte gonioskopisch zu graulichen Poren im Bereich des Trabekelwerks. Es zeigte sich, daß die Effekte mit Hilfe der Quarzfaser ohne Schwierigkeit sehr präzise dort gesetzt werden konnten, wo sie die Verbindung zwischen Kammerwasser und Schlemmschem Kanal herstellen sollten. Die histologische Untersuchung des Kammerwinkels dieses Auges bestätigte die exakte Lage der Effekte im Bereich des Trabekelwerks (Abb. 4 und $4 a$ ).

\section{Diskussion}

Die ophthalmologische Chirurgie macht sich seit einiger Zeit die Möglichkeiten der Gewebedurchtrennung durch Ablation mit dem Excimer-Laser zunutze. Insbesondere wird diese "Schnittechnik" auf dem Gebiet der refraktiven Hornhautchirurgie benutzt. Inzwischen sind auch Berichte erschienen über eine Trepanation von innen - d.h. mit einer Quarzfaser wird eine Perforation von innen im Bereich des Limbus durchgeführt - so daß das Kammerwasser, wie nach einer fistulierenden Operation, unter die Bindehaut abgeführt werden kann (Müller-

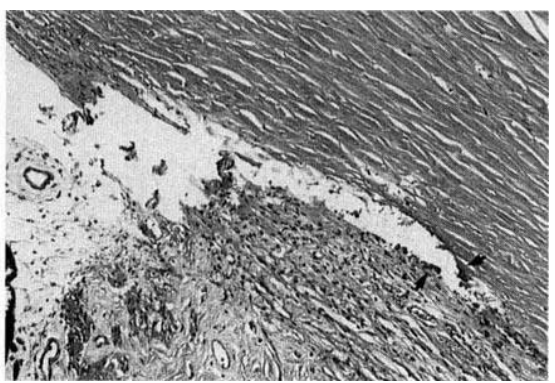

Abb. 2a Vergrößerter Ausschnit aus Abb. 2. Am Ende des Cyclodialysespaltes erkennt man eine sehr geringfügige Basophilie als Hinweis auf einen thermischen Effekt (Pfeile). (Hämatoxylin-Eosin, $40 \times$ ) 


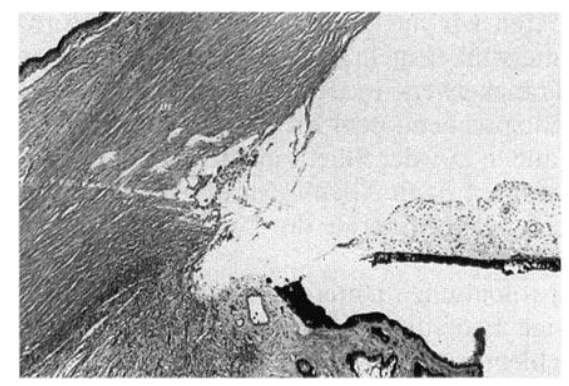

Abb. 3 Ablation der Iriswurzel des vorderen Anteils des Ciliarköpers und teilweise Ablation des Trabekelwerks. Gearbeitet wurde mit Wellenlänge $308 \mathrm{~nm}$, Pulslänge 20 ns, Energie 1,0 $\mathrm{mJ}$

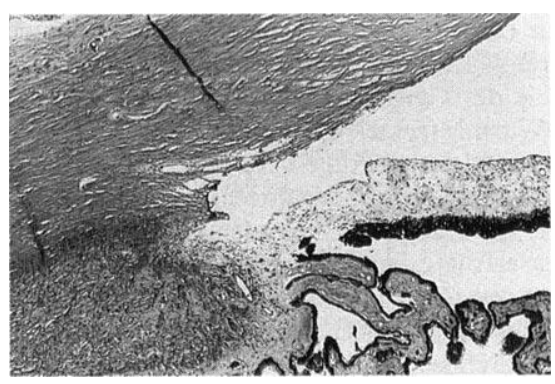

Abb. 4 Präzise Ablation des Trabekelwerks mit offener Verbindung zwischen Vorderkammer und Schlemmschem Kanal. Gearbeitet wurde mit Wellenlange $308 \mathrm{~nm}$, Pulslänge 20 ns, Energie 1,0-1,5 mJ. (Hämatoxylin-Eosin, 16x)

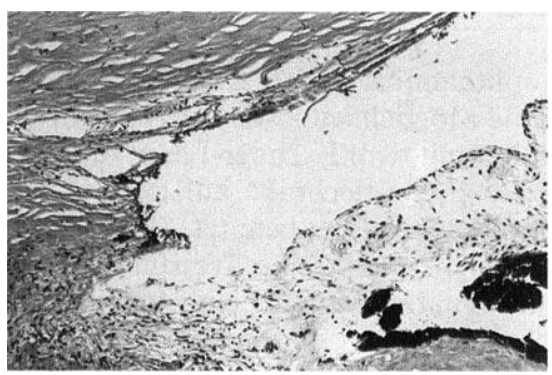

Abb. 4a Vergrößerter Ausschnitt aus Abb. 4

Einzelne Fasern des vorderen Trabekelwerks sind noch zu erkennen. Der hintere Anteil ist verschwunden. Am Rand des Ablationsbereiches erkennt man eine minimale basophile Zone als Ausdruck des thermischen Effekts (Pfeile). (HämatoxylinEosin, 40x)

Stolzenburg und Müller, 1989). Wetzel et al. (1989) und March et al. (1985 und 1987) arbeiteten mit einem Neodym-YAG-Laser bzw. mit Argon- und Neodym-YAG-Laser. Weitere Erfahrungen bestehen mit dieser Methode noch nicht.

Epstein et al. (1985) führten Trabekulopunkturen mit dem Neodym-YAG-Laser durch, beobachteten aber trotz der hohen angewandten Energien in allen Fällen im Laufe der Zeit einen gonioskopischen Verschluß der Punktionsstellen. Sie halten diese Methode am ehesten für juvenile und congenitale Glaukome geeignet. Die An- wendung des Neodym-YAG-Lasers hat gegenüber dem von uns vorgeschlagenen Weg den Vorteil, daß man das Auge nicht zu eröffnen braucht, weil die Koagulation über ein Kontaktglas möglich ist. Wie aus der Arbeit von Dutton et al. (1987) aber zu entnehmen ist, muß bei diesem Vorgehen sehr genau gezielt werden, um den hinteren Anteil des Trabekelwerks wirklich mit dem Laserstrahl zu treffen. Melamed et al. (1987) untersuchten, ob eine punktuelle oder konfluierende Koagulation erfolgversprechend sei.

Del Priore et al. (1988) benutzten den Neodym-YAG-Laser, um bei 61 Patienten (79 Augen) das Trabekelwerk zu eröffnen. Die Autoren berichten über eine Drucksenkung bei $46 \%$ ihrer Patienten nach einem Jahr.

Die Eröffnung des Trabekelwerks mit dem Excimer-Laser ist infolge der Ablation des Gewebes schonender und mit der Quarzfaser gezielter möglich. Das Trabekelwerk wird nicht mit einer Mikroexplosion zerrissen, sondern löst sich unter dem Excimer-Laser-Licht auf. Entsprechend unserer bereits 1973 (Vogel und Schildberg) erklärten Absicht, versuchen wir mit dem Excimer-Laser und einer Quarzfaser unser Ziel zu erreichen: den Hauptabflußwiderstand beim Glaucoma chronicum simplex zu beseitigen. Die hier mitgeteilten Untersuchungen wurden an Schweine- und an enukleierten Augen durchgeführt. Wir können daher noch keine Auskunft darüber geben, ob es tatsächlich zu der angestrebten Drucksenkung kommt. Die durchgeführten Experimente scheinen uns aber ein sinnvoller Ansatz zu sein, dieses Ziel zu erreichen. Da die Ablation des Gewebes nur zu einer sehr umschriebenen, minimalen Nekrotisierung führt, weil sich das Gewebe unter dem Excimer-Laserstrahl vollständig auflöst, erwarten wir keine nennenswerte entzündliche Reaktion auf diesen Eingriff und damit ein Offenbleiben der Verbindungen zwischen Vorderkammer und Schlemmschem Kanal. Unsere Untersuchungen lassen es möglich erscheinen, neben der Eröffnung des Trabekelwerks auch Cyclodialysen und basale Iridektomien mit dem ExcimerLaser durchzuführen.

\section{Literatur}

1 Bill, A., O. Mälpea, T. Hamanaka: Aspekte der Kammerwasserdrajnage über dem Schlemmschen Kanal. Klin. Mbl. Augenhk. 195 (1989) 277-289

Del Priore, L. V., A. L. Robin, I. P. Pollak: Longterm follow-up of neodymium: YAG laser angle surgery for open-angle glaucoma. Ophthaimology 95 (1988) 277-281

Dutton, G. N., D. Allan, S. A. Cameron: Aiming criteria for neodymium-YAG laser trabeculotomy. A clinico-pathological study. Eye 1 (1987) 401-405

${ }^{4}$ Epstein, D. L., S. Melamed, C. A. Puliafito, R. F. Steinert: Neodymium: YAG laser trabeculopuncture in open-angle glaucoma. Ophthalmology 92 (1985) $931-937$

Hager, H.: Besondere mikrochirurgische Eingriffe: Erste Erfahrungen mit dem Argon Laser Gerät 800. Klin. Mbl. Augenhk. 162 (1973) 437450

"Krasnov, M. M.: Laser puncture of anterior chamber angle in claucoma. Am. J. Ophthal. 75 (1973) 674-678

March, W. F., T. Gherezghiher, M. C. Koss, R. P. Shaver, W. D. Heath, R. D. Nordquist: Histologic study of a neodymium-YAG laser sclerostomy. Arch. Ophthalmol. (Chicago) 103 (1985) 860-863

${ }^{8}$ March, W. F., T. Gherezghiher, R. P. Shaver, M. C. Koss, R. E. Nordquist: Safety of high-energy neodymium: YAG laser pulses in YAG sclerostomy. Laser Surg. Med. 6 (1987) 584-587 
${ }^{9}$ Melamed, S., M. A. Latina, D. L. Epstein: Neodymium: YAG laser trabeculopuncture in juvenile open-angle glaucoma. Ophthalmology 94 (1987) 163-170

${ }^{10}$ Muller-Stolzenburg, N., G. J. Müller: Transmission of $308 \mathrm{~nm}$ excimer laser radiation for ophthalmic microsurgery. Medical, technical and safety aspects. Biomed. Technik 34 (1989) 131-138

"Vogel, M., P. Schildberg: Histologische Frühergebnisse nach experimenteller Lasertrabekulopunktur. Klin. Mbl. Augenhk. 163 (1973) 353-358

12 Vogel, M., S. Ecker, G. Cieplik, G. Bernhardt: Lasertrabekuloplastik bei Glaucoma chronicum simplex. Ergebnisse einer prospektiven, klinischen Studie. Klin. Mbl. Augenhk. 193 (1988) 8-15

13 Wetzel, W., G. Duncker, C. Schumacher, W. Dölle: Laser-Sklerostomie: Alternative zu bekannten operativen fistulierenden Verfahren. Experimentelle Untersuchungen. Klin. Mbl. Augenhk. 194 (1989) 170172

14 Wise, J. B., S. L. Witter: Argon laser therapy of open-angle glaucoma. A pilot study. Arch. Ophthal. (Chicago) 97 (1979) 319-322
Manuskript erstmals eingereicht 5. 2. 1990, zur Publikation in der vorliegenden Form angenommen 26. 2. 1990.

\section{Prof. Dr. M. Vogel}

Leiter der Abteilung Augenheilkunde Universitäts-Augenklinik Göttingen Robert-Koch-Str. 40

3400 Göttingen 